\title{
Subdural hematomas in boxing: the spectrum of consequences
}

\author{
Vincent J. Miele, M.D., Julian E. Bailes, M.D., Robert C. Cantu, M.D., \\ and Craig H. Rabb, M.D.
}

\author{
Department of Neurosurgery, West Virginia University School of Medicine, Morgantown, \\ West Virginia; Department of Surgery, Emerson Hospital, Concord, Massachusetts; \\ and Department of Neurosurgery, University of Colorado School of Medicine, \\ Denver Health Medical Center, Denver, Colorado
}

\begin{abstract}
$\checkmark$ Boxing is a violent sport in which every participant accepts the risk of brain damage or death. This sport has been linked to acute neurological injury and chronic brain damage. The most common life-threatening injury encountered by its participants is subdural hematoma (SDH), and the most feared consequence of chronic insult to the nervous system is dementia pugilistica, or punch drunkenness. Although advances in imaging and neuropsychological testing have improved our ability to diagnose these injuries, the unprecedented sensitivity and wide availability of these modalities have increased the detection of mild cognitive impairment and small, asymptomatic imaging abnormalities. The question has thus been raised as to where on the spectrum of these injuries an athlete should be permanently banned from the sport.

In this report the authors describe six boxers who were evaluated for SDH sustained during participation in the sport, and who experienced remarkably different outcomes. Their presentations, clinical courses, and boxing careers are detailed. The athletes ranged in age from 24 to 55 years at the time of injury. Two were female and four were male; half of them were amateurs and half were professionals. Treatments ranged from observation only to decompressive craniectomy. Two of the athletes were allowed to participate in the sport after their injury (one following a lengthy legal battle), with no known sequelae. One boxer died within 48 hours of her injury and at least two suffered permanent neurological deficits. In a third, dementia pugilistica was diagnosed 40 years later, and the man died while institutionalized.
\end{abstract}

KeY WORDS • boxing • head injury • subdural hematoma

$\mathrm{B}$ OXING is a violent sport in which athletes accept the risk of brain damage or death. The most common life-threatening injury encountered by its participants is SDH, and the most feared consequence of chronic insult to the nervous system is dementia pugilistica, or punch drunkenness. ${ }^{1,12,13,18}$ Traditionally, if during testing a boxer was found to have suffered these injuries, the individual was permanently excluded from further participation in the sport. Although recent advances in neuropsychological testing have improved our ability to diagnose mild cognitive impairment, the unprecedented sensitivity and wide availability of new imaging modalities have increased the detection of small, asymptomatic abnormalities. The following question has thus been raised: where on the spectrum of these injuries should a boxer be permanently banned from the sport? In this study we describe six athletes who suffered an SDH while participating in the sport of boxing, and we relate their various outcomes, including sequelae, from return to competition.

\footnotetext{
Abbreviations used in this paper: $\mathrm{CT}=$ computed tomography; GCS = Glasgow Coma Scale; $\mathrm{MR}=$ magnetic resonance imaging; $\mathrm{SDH}=$ subdural hematoma; $\mathrm{TBI}=$ traumatic brain injury.
}

\section{Illustrative Cases}

\section{Boxers in Whom SDH With Minimal Symptomatology Was Found}

Case 1. This 27-year-old amateur boxer, who had been training for 2 years and had participated in six amateur matches, was seen in consultation. Eight months previously, in the third round of an amateur bout, he received four hard blows to the head and went down to one knee, causing the match to be stopped. Later that evening at a local hospital, a CT scan of the head was obtained on which a small (5-mm) right frontal SDH with no mass effect (Fig. 1) was demonstrated. The patient was neurologically intact, with a GCS score of 15. A second CT scan performed within 24 hours confirmed the presence of a small SDH with no mass effect. The next morning the patient was discharged and was not seen by a physician until 8 months later, when he presented for clearance to resume boxing. $\mathrm{He}$ stated that 2 months after the initial incident, feeling well, he had resumed boxing and had been sparring regularly for the last 6 months. At the time of the examination he was asymptomatic and had been vigorously training for 6 months. Results of his neurological examination, including detailed mental status, were all within normal limits. An MR image of his brain demonstrated no evidence of 




FIG. 1. Case 1. Initial CT scan demonstrating a small (5-mm) right frontal SDH with no mass effect.

residual hematoma. Based on the normal results on MR imaging and neurological examination and because there were no postconcussion symptoms, the patient was cleared to continue boxing and has been doing so with no further injury.

Case 2. This 31-year-old superheavyweight Golden Gloves champion with 29 professional wins (including 25 knockouts) was looking forward to a bout with a multimillion dollar payday when two SDHs were discovered following a unanimous 10-round decision victory. During this match, he had been dominated in the eighth round and knocked down once in the ninth and twice in the 10th. Nonetheless, his margin of victory was 94 to 93 on all three judges' scorecards due to his command of the bout before the eighth round. Following the match, the boxer was unable to remember the final round and events that occurred immediately after the bout. Over the next several weeks, he began to complain of headaches and was reportedly incoherent at times. Because of this, the athlete agreed to be examined by a physician, and MR imaging was performed. This study revealed two small SDHs that were causing no mass effect or midline shift (Fig. 2).

For the next year, the athlete did not participate in boxing. Repeated MR imaging was performed that suggested complete resolution of the abnormalities. The boxer underwent multiple neurological examinations, on which no residual damage was found. Nevertheless, his state's athletic commission (the sanctioning body for all boxing in that location), in keeping with its policy, unanimously voted to suspend the athlete indefinitely from future participation. Due to federal legislation (the Muhammad Ali Boxing Reform Act) mandating that all states honor the suspensions and bans issued by any other, this essentially banned the boxer from participation anywhere in the US. The basis of the commission's position that the athlete was unfit to box was the theory that because he had already suffered one SDH, he was at greater risk for another. The athlete appealed this decision, and it was overturned by the Nevada state court system. He returned to competition in Puerto Rico and won an eighth-round knockout in his return debut. After this return match, a new MR image revealed no recurrence of hemorrhage.

\section{Boxers in Whom SDH Resulting in Life-Threatening Mass Effect Occurred}

Case 3. This 34-year-old female boxer wearing headgear collapsed during an amateur match. Her muscles immediately became flaccid and she was comatose for 15 minutes in the ring, during which time she vomited and agonal respirations developed. After an unsuccessful intubation attempt, ventilation was maintained with bag and mask, and she was then transported to a medical facility 10 minutes from the venue. On arrival her GCS score was 3 , and both pupils were fixed and dilated. On admission CT scans a large right-sided SDH was revealed (Fig. 3 left), with complete obliteration of the basal cisterns (Fig. 3 center). She was taken immediately to the operating room, where SDH evacuation and a large craniectomy were performed. Intraoperatively, the patient exhibited signs of coagulopathy and diabetes insipidus. She showed no improvement in neurological status postoperatively, and a postoperative CT scan demonstrated widespread

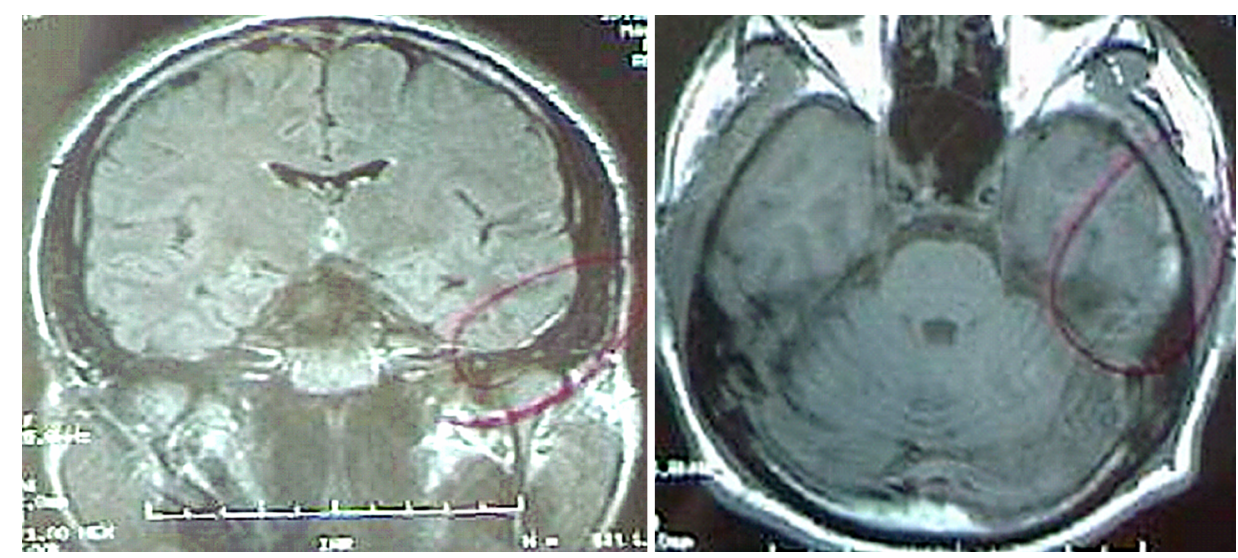

FIG. 2. Case 2. Initial coronal (left) and axial (right) $\mathrm{T}_{1}$-weighted MR images demonstrating a thin left temporal SDH. 

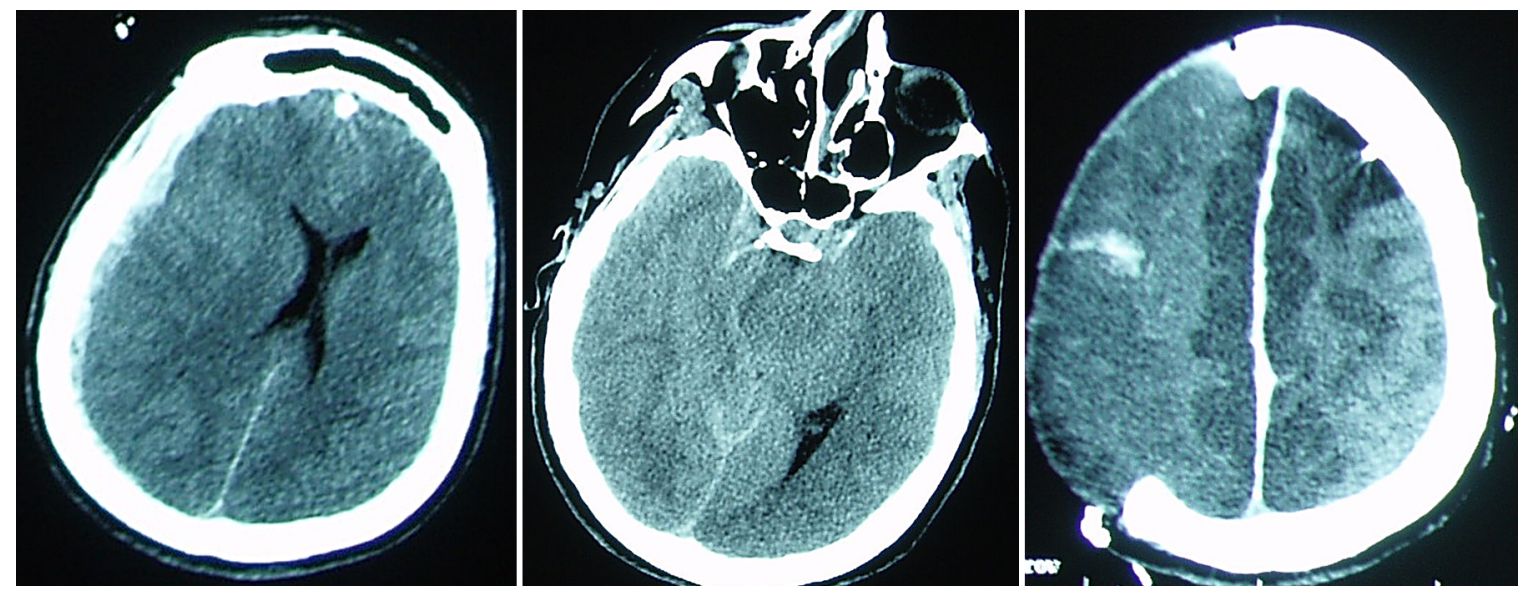

FIG. 3. Case 3. Left: Preoperative CT scan revealing right hemispheric hyperdensity with a midline shift. Center: Preoperative CT scan demonstrating complete effacement of the basal cisterns. Right: Postoperative CT scan demonstrating a large craniectomy defect and multiple hypodense areas with the appearance of infarction.

bilateral cerebral infarctions (Fig. 3 right). The next day we determined that she fit the criteria for brain death.

Case 4. This 55-year-old man was struck multiple times about the head while boxing. He fell to the ground, attempted to grab the ropes, vomited, and collapsed. On examination by paramedics, he was comatose and his left pupil was fixed and dilated. An oropharyngeal airway was placed and he was unresponsive for approximately 30 minutes before transport to a medical facility, where he arrived with a GCS score of 3. An admission CT scan demonstrated a large left-sided SDH (Fig. 4), and the patient was taken immediately to the operating room,



FIG. 4. Case 4. Preoperative scan demonstrating left hemispheric hyperdensity with a significant mass effect and midline shift. where a large craniectomy and evacuation of the hematoma were performed. Postoperatively, the patient exhibited left hemiplegia, presumably the result of a Kernohan notch phenomenon. Over the next 2 months he slowly improved and began to follow commands. He was discharged to a skilled nursing facility and has begun to recover function on his left side.

Case 5. This 24-year-old professional boxer presented with headache and nausea of increasing intensity the morning after a bout. She had begun boxing approximately 1 year prior to presentation, and despite having participated in only two sanctioned matches, she was elected National Boxer of the Year for her organization. Two weeks before presentation, the patient was matched with a $120-\mathrm{kg}$ male opponent for sparring and was knocked down with a right hook when the male boxer became frustrated. There was no loss of consciousness and she was alert and oriented immediately after the incident. This knockdown ended the sparring session, and only minutes afterward the patient experienced a global headache, which increased in intensity over the next week. Two weeks after the sparring session, although she was still symptomatic, the patient was allowed to participate in a contest, which she stopped voluntarily during the second round when her headache became intolerable. She did not sustain any significant trauma to the head during that event. She presented to the emergency room the next morning $(\sim 12$ hours after the bout) when her headache pain became progressive and unbearable.

On arrival at the hospital, the patient had a GCS score of 15 , and she reported a severe headache and nausea. Results of the neurological examination and coagulation studies were within normal limits. An admission CT scan revealed a large acute-on-chronic SDH over the left cerebral hemisphere that was causing a large left-to-right midline shift with partial effacement of the ipsilateral basilar cisterns and lateral ventricle (Fig. 5). A craniotomy was performed, and approximately 300 to $400 \mathrm{ml}$ of blood under high pressure and a hematoma containing materials from different stages of its evolution was discovered on opening the dura mater. After evacuation of the clot and removal of associated membranes, there was immediate reexpansion of the un- 
derlying parenchyma. The patient tolerated the procedure well and awakened immediately after surgery with a GCS score of 15 and no neurological deficits.

One month postsurgery, the patient reported persistent neuropsychological difficulties. These included subjective memory, concentration, and language problems. She described frequent loss of her train of thought, forgetfulness, blurry vision, word-finding difficulties, slurring of speech, and spelling problems. In addition, she reported increasing levels of depression and anxiety. A neuropsychological evaluation was performed and revealed several objective deficits. Confrontation naming was in the low to average range and below expectations, given her educational level. The patient also performed in the low to average range on tests of memory for verbal information and tasks requiring manipulation of learned information. Overall, she displayed a pattern of problems with memory, concentration, and speed of processing that would be consistent with acute TBI. The patient did not return to the sport of boxing and is currently undergoing neuropsychological rehabilitation.

\section{Subdural Hematoma Resulting in Dementia Pugilistica}

Case 6. This 72-year-old former professional boxer was admitted for a dementia workup. During his career, he had won a Golden Gloves title and was an early opponent of Muhammad Ali (then known as Cassius Clay) in professional boxing in 1960. In 1962, following a 10-round professional bout, he became unresponsive and was found to have suffered an SDH. Two surgeries were performed to evacuate the clot and he remained in a coma for 9 days. He slowly recovered, to the point that he could continue his duties as a police chief in a small town. His career was ended by this injury when he was 32 years of age, and he retired with a record of 15 wins and 15 losses.

After his retirement, this boxer demonstrated a decline in his behavior and memory, with increasingly frequent falls and altered mental status. He had difficulty with all activities of daily living and began wandering away from home inappropriately. On neurological examination he demonstrated poor remote memory as well as disorientation to date, place, and situation. Although he was able to perform simple math calculations, he could not name the president of the US and was dysarthric. He was not able to follow complex one-step commands and was ataxic, with a wide-based, shuffling gait. Brain imaging studies (CT and MR imaging) demonstrated diffuse atrophy, enlarged ventricles, and encephalomalacia in the frontal and occipital lobes (Fig. 6 left). Fluid-attenuated inversion-recovery MR images demonstrated periventricular deep white matter disease (Fig. 6 right). The ventricles were enlarged and there was diffuse encephalomalacia. These changes were consistent with previous TBI. No diffusion restrictions were seen. An electroencephalography study demonstrated mild generalized abnormalities. We diagnosed dementia pugilistica in this patient, and he received follow-up care in a dementia and memory clinic. However, because of his combativeness and confusion, the patient required placement in a long-term care facility and died there at the age of 75 years.



FIG. 5. Case 5. Preoperative CT scan demonstrating a large heterogeneous subdural fluid collection over the left cerebral hemisphere causing a midline shift and partial effacement of the ipsilateral lateral ventricle. Note the multiple densities of blood, a finding consistent with an acute-on-chronic SDH.

\section{Discussion}

\section{Subdural Hematomas in Boxing}

Subdural hematomas are the most common form of sports-related intracranial bleeding. They are more common than epidural hematomas and account for the majority of lethal brain injuries seen in both organized and recreational athletic activities. ${ }^{1,5}$ It is important to understand that SDHs in athletes are dissimilar to those commonly seen in the elderly and in many nonathletes who are trauma victims. The athlete usually does not have the large potential subdural space that an elderly patient possesses, and therefore a mass effect and increases in intracranial pressure can occur with greater rapidity. In addition to injury from the mass effect of blood beneath the dura mater, there is often significant associated damage (contusion or edema) to the underlying brain due to multiple (sometimes hundreds) of blows. Whereas the incidence of subclinical intracranial hemorrhage in boxers is unknown, acute SDHs and associated cerebral edema are the leading cause of boxing-related death. ${ }^{21}$ As demonstrated in our cases, boxers who sustain an SDH may immediately become unconscious and/or suffer focal neurological deficits, or symptomatology may develop insidiously over days or even weeks. As demonstrated in the presented cases, the sequelae range from acute neurological complications to the chronic deteriorating process of dementia pugilistica or death. 



FIG. 6. Case 6. Left: Admission CT scan demonstrating changes consistent with an earlier right frontotemporal craniectomy. Also evident are large hypodense areas involving portions of the right frontal, occipital, and temporal lobes as well as the left temporal lobe. Additionally, asymmetrical expansion of the right lateral ventricle with periventricular hypodensity, predominantly at the right lateral ventricle, is apparent. Right: A fluid-attenuated inversion-recovery MR image revealing dilated lateral ventricles. Periventricular high-intensity signal changes are evident. Subcortical highintensity signal changes are scattered within both hemispheres and are especially prominent in the right medial subfrontal area and the occipital region. Right temporal lobe atrophy is also marked. No diffusion abnormalities indicating acute ischemic events were identified. Subcortical white matter changes and focal changes consistent with probable earlier ischemic and/or traumatic injury are evident.

Although CT and MR imaging studies are extremely useful tools for the evaluation of boxers for intracranial injury, imaging studies also may be used as evidence barring these athletes from future participation in the sport. Thus, at times there may be an incentive for them to refuse or avoid such tests. Although no evidence has been published supporting the suggestion that a boxer who has suffered an intracranial hemorrhage is at any increased risk of such events in the future, governing bodies in the sport may consider this finding to be sufficient evidence to bar the fighter from future participation. Whereas athletic commissions want to be cautious, athletes on the other hand aspire to win championships and million-dollar paychecks. They may not be easily dissuaded by imaging findings, especially when symptoms do not exist or have resolved. Athletes who perceive that they will be banned from the sport will be less likely to seek diagnosis and treatment.

There is little in the medical literature to guide us, although the return of athletes to the sport of professional boxing at the elite level after discovery of intracranial abnormalities has been documented. ${ }^{9-11}$ One such individual was Marco Antonio Barrera, a world-class boxer in the featherweight division, who began to complain of headaches in 1995. In 1997, a neurosurgeon in Mexico City examined him and discovered a cavernous angioma, which was removed in an operation performed in August 1997. After the surgery, Barrera participated in 16 matches, until his medical history became public just weeks before a scheduled bout in San Antonio, Texas. After numerous examinations and imaging studies, the Texas Boxing Commission gave Barrera a green light to participate.
If an athlete is barred from competition in the US, he or she may choose to participate outside of the country. This occurred recently with a boxer, Edwin Valero, who was suspended for an intracranial abnormality secondary to brain trauma discovered on MR imaging. Venezuelan Boxing Federation medical records indicate that an SDH was detected in Valero and was removed using a drainage procedure. The athlete was suspended by the New York State Athletic Commission and the Association of Boxing Commissions from competing in the US ${ }^{6,9}$ Nevertheless, after being cleared by doctors in Argentina, Valero has resumed his career by boxing outside the US, without obvious consequences. Another example of the incentive for a boxing jurisdiction to be more lenient or tolerant of an injury is demonstrated in our Case 2, in which the athlete chose his return venue in part based on its support of his return to boxing. Additionally, the athletes presented in our Cases 1 and 2 have returned to competition after a diagnosis of $\mathrm{SDH}$, with no recurrent symptoms.

Although these athletes who have returned to competition have not suffered a recurrence of intracranial hemorrhage, much less is known about the long-term effects of their injuries. Chronic neurological injuries from boxing tend to have an insidious onset and often present and continue to progress after retirement. ${ }^{14}$ The occurrence of chronic neurological injuries in a retired National Football League player has recently been suggested based on autopsy findings. ${ }^{20}$ In several studies investigators have demonstrated that seemingly minor head injuries are often associated with measurable long-term consequences. ${ }^{8} \mathrm{Re}$ petitive blows to the head result in axon damage and neu- 
ron death, and whereas a limited injury may not produce symptoms, as their effects accumulate, so does the incidence of clinical abnormalities. ${ }^{15,17}$

The influence of TBI on neuropsychological functioning has been demonstrated in several studies..$^{2-4,719}$ Areas most significantly affected include working memory/attention and speed of processing, which depend on the athlete's ability to be attentive and to concentrate. ${ }^{7}$ The risk of chronic neurological injury probably increases in a cumulative manner with every punch absorbed; therefore, professional boxers, especially those who have participated in the sport for more than 10 years, are particularly at risk. Although gross abnormalities on neurological examination can be observed in the most severe cases of boxing-related encephalopathy, and include cerebellar, extrapyramidal, and intellectual impairments, less easily identifiable changes in neurocognitive function are believed to occur more often. ${ }^{16}$ In the cases presented, two athletes who did not return to competition retired because of objectively diagnosed long-term neuropsychological abnormalities.

\section{Conclusions}

Acute SDHs sustained while participating in the sport of boxing can result in a wide variety of sequelae, from mild symptomatology such as headaches to focal neurological deficits and death. This sport also carries the risk of chronic neurological injury. The spectrum of adverse events is illustrated by the cases discussed. Although preparticipation medical and neuroimaging assessments have resulted in the detection of small amounts of intracranial hemorrhage and early and potentially presymptomatic cases of chronic TBI, significant controversy exists over how to use the information. Enforcing well-intentioned rules that are not substantiated by scientific data could encourage a "don't ask, don't tell" atmosphere when it comes to brain injuries, which is the exact opposite of the rules' intent. Should a central governing body be given the authority to decide an athlete's acceptable level of risk without the decision being evidence-based? It is difficult to defend ending a professional career based on the argument that the athlete is at an increased risk of future injury when no data exist to support this claim. Although chronic neurological injuries have not garnered the same amount of attention as their acute counterpart, chronic injuries can potentially affect a much larger number of participants. More research is essential to objectify the reasoning behind possibly ending an athlete's career and livelihood.

\section{References}

1. Bailes JE, Cantu RC: Head injury in athletes. Neurosurgery 48:26-46, 2001

2. Binder LM: A review of mild head trauma. Part II: clinical implications. J Clin Exp Neuropsychol 19:432-457, 1997
3. Binder LM, Kelly MP, Villanueva MR, Winslow MM: Motivation and neuropsychological test performance following mild head injury. J Clin Exp Neuropsychol 25:420-430, 2003

4. Binder LM, Rohling ML, Larrabee J: A review of mild head trauma. Part I: meta-analytic review of neuropsychological studies. J Clin Exp Neuropsychol 19:421-431, 1997

5. Cantu RC, Mueller FO: Brain injury-related fatalities in American football, 1945-1999. Neurosurgery 52:846-853, 2003

6. Contursi S: Valero (14-0, 14 KOs) fights despite U.S. ban, in ESPN News (http://sports.espn.go.com/sports/boxing/news/ story?id=211597) [Accessed 18 August 2006]

7. Frencham KAR, Fox AM, Maybery MT: Neuropsychological studies of mild traumatic brain injury: a meta-analytic review of research since 1995. J Clin Exp Neuropsychol 27:334-351, 2005

8. Guskiewicz KM, Marshall SW, Bailes J, McCrea M, Cantu RC, Randolph C, et al: Association between recurrent concussion and late-life cognitive impairment in retired professional football players. Neurosurgery 57:719-726, 2005

9. Hauser T: Boxing's medical mess, in Bernath C (ed): Secondsout.com. (http://www.secondsout.com/usa/colhauser. $\mathrm{cfm}$ ?ccs=208\&cs=13484) [Accessed 18 August 2006]

10. Hauser T: The rumors about Joe Mesi, in Bernath $C$ (ed): Secondsout.com. (http://www.secondsout.com/usa/colhauser. cfm?ccs=208\&cs=14736) [Accessed 18 August 2006]

11. Iole K: Barrera: 'The truth has set him free,' in Tiger Boxing. (http://www.tigerboxing.com/articles/index.php?aid=1001244 770) [Accessed 18 August 2006]

12. Jordan B: Sports injuries, in Proceedings of the Mild Brain Injury in Sports Summit. Dallas, Texas: National Athletic Trainers' Association, Inc., 1994, pp 43-46

13. Jordan BD: Chronic traumatic brain injury associated with boxing. Semin Neurol 20:179-186, 2000

14. Jordan BD: Neurologic aspects of boxing. Arch Neurol 44: 453-459, 1987

15. Martland HS: Punch drunk. JAMA 91:1103-1107, 1928

16. Mendez MF: The neuropsychiatric aspects of boxing. Int J Psychiatry Med 25:249-262, 1995

17. Miele VJ, Bailes JE: Objectifying when to halt a boxing match: a video analysis of fatalities. Neurosurgery, in press, 2006

18. Miele VJ, Bailes JE, Voelker JL: Boxing and the neurosurgeon. Clin Neurosurg 49:396-406, 2002

19. Miele VJ, Carson L, Carr A, Bailes JE: Acute on chronic subdural hematoma in a female boxer: a case report. Med Sci Sports Exerc 36:1852-1855, 2004

20. Omalu BI, DeKosky ST, Minster RL, Kamboh MI, Hamilton RL, Wecht CH: Chronic traumatic encephalopathy in a National Football League player. Neurosurgery 57:128-134, 2005

21. Unterharnscheidt F: About boxing: review of historical and medical aspects. Tex Rep Biol Med 28:421-495, 1970

Manuscript received August 7, 2006.

Accepted in final form September 7, 2006.

Address reprint requests to: Vincent J. Miele, M.D., Department of Neurosurgery, West Virginia University School of Medicine, P.O. Box 9183, Morgantown, West Virginia 26506-9183. email: lesvin@adelphia.net. 